\title{
Genetic Interference Analysis Reveals that Both 3-Hydroxybenzoic Acid and 4-Hydroxybenzoic Acid Are Involved in Xanthomonadin Biosynthesis in the Phytopathogen Xanthomonas campestris pv. campestris
}

\author{
Xue-Qiang Cao, ${ }^{1}$ Xing-Yu Ouyang, ${ }^{1}$ Bo Chen, ${ }^{1}$ Kai Song, ${ }^{1}$ Lian Zhou, ${ }^{1}$ Bo-Le Jiang, ${ }^{2}$ Ji-Liang Tang, ${ }^{2}$ Guanghai Ji, ${ }^{3}$ \\ Alan R. Poplawsky, ${ }^{4}$ and Ya-Wen $\mathrm{He}^{1, \dagger}$ \\ ${ }^{1}$ State Key Laboratory of Microbial Metabolism, Joint International Research Laboratory of Metabolic \& Developmental Sciences, School of \\ Life Sciences \& Biotechnology, Shanghai Jiao Tong University, Shanghai 200240, China \\ ${ }^{2}$ State Key Laboratory for Conservation and Utilization of Subtropical Agro-bioresources and College of Life Science and Technology, Guangxi \\ University, Nanning 530004, China \\ ${ }^{3}$ Key Laboratory of Agriculture Biodiversity for Plant Disease Management, Ministry of Education, College of Plant Protection, Yunnan \\ Agricultural University, Kunming 650201, China \\ ${ }^{4}$ Department of Entomology, Plant Pathology and Nematology, University of Idaho, Moscow, ID 83844, U.S.A. \\ Accepted for publication 12 October 2019.
}

\begin{abstract}
A characteristic feature of phytopathogenic Xanthomonas bacteria is the production of yellow membrane-bound pigments called xanthomonadins. Previous studies showed that 3-hydroxybenzoic acid (3-HBA) was a xanthomonadin biosynthetic intermediate and also, that it had a signaling role. The question of whether the structural isomers 4-HBA and 2-HBA (salicylic acid) have any role in xanthomonadin biosynthesis remained unclear. In this study, we have selectively eliminated 3-HBA, 4HBA, or the production of both by expression of the $m h b$, pobA, and $p c h A B$ gene clusters in the Xanthomonas campestris pv. campestris strain $\mathrm{XC1}$. The resulting strains were different in pigmentation, virulence factor production, and virulence. These results suggest that both 3-HBA and 4-HBA are involved in xanthomonadin biosynthesis. When both 3HBA and 4-HBA are present, X. campestris pv. campestris prefers 3-HBA for Xanthomonadin-A biosynthesis; the 3-HBA-derived Xanthomonadin-
\end{abstract}

ABSTRACT

Members of the Gram-negative genus Xanthomonas cause disease in a large number of plants worldwide and are responsible for serious losses in agricultural production (Hayward 1993; Leyns et al. 1984). Xanthomonas campestris pv. campestris, the causal agent of black rot of crucifers, represents a model organism for the study of pathogenicity in phytopathogenic bacteria (Mansfield et al. 2012). A characteristic feature of Xanthomonas bacteria is the production of yellow membrane-bound pigments called xanthomonadins (Stephens and Starr 1963). Several studies have indicated that xanthomonadins protect bacterial cells from photooxidative damage (Jenkins and Starr 1982; Poplawsky et al. 2000; Rajagopal et al. 1997). In addition, xanthomonadin extracts from Xanthomonas oryzae pv. oryzae displayed antioxidant activity by inhibiting lipid peroxidation in liposomes (Rajagopal et al. 1997). Furthermore, Poplawsky and Chun (1997) and Poplawsky et al. (2000) found that xanthomonadin-deficient $X$. campestris pv. campestris

†Corresponding author: Y.-W. He; yawenhe@sjtu.edu.cn

Funding: This work was supported by National Natural Science Foundation of China grants 31471743 (to Y.-W. He) and 31772121 (to Y.-W. He).

X.-Q. Cao and X.-Y. Ouyang contributed equally to this work.

*The $e$-Xtra logo stands for "electronic extra" and indicates that supplementary materials are published online.

The author(s) declare no conflict of interest.

(C) 2020 The American Phytopathological Society
A was predominant over the 4-HBA-derived xanthomonadin in the wildtype strain XC1. If 3-HBA is not present, then 4-HBA is used for biosynthesis of a structurally uncharacterized Xanthomonadin-B. Salicylic acid had no effect on xanthomonadin biosynthesis. Interference with 3-HBA and 4-HBA biosynthesis also affected X. campestris pv. campestris virulence factor production and reduced virulence in cabbage and Chinese radish. These findings add to our understanding of xanthomonadin biosynthetic mechanisms and further help to elucidate the biological roles of xanthomonadins in X. campestris pv. campestris adaptation and virulence in host plants.

Keywords: bacteriology, genetics and resistance, salicylic acid, Xanthomonas campestris, xanthomonadin, 3-hydroxybenzoic acid, 4-hydroxybenzoic acid

mutant was reduced in the bacterial epiphytic survival rate and host infection following exposure to high-intensity natural light. Our previous results showed that deletion of $x a n B 2$, which is essential for xanthomonadin biosynthesis, led to reduced virulence in radish plants (He et al. 2011). Collectively, these findings suggest that xanthomonadins play important roles in maintaining ecological fitness by protecting the Xanthomonas bacteria against photooxidative and peroxidative stress.

Xanthomonadins were found to be located on the bacterial outer membrane (Dianese and Schaad 1982; Stephens and Starr 1963). Structural analysis of xanthomonadin isobutyl esters by X-ray crystallographic and mass spectrum methods demonstrated that they represent a unique group of halogenated aryl-polyene pigments (Andrewes et al. 1973, 1976). One Xanthomonas species usually produces several xanthomonadins, which differ in methylation and bromination patterns at the aryl ring and also, polyene chain length (Starr et al. 1977). Our latest data showed that there is no methylation at the aryl ring in $\mathrm{XC} 1$ xanthomonadins (Cao et al. 2018). Aririatu and Kester (1985) isolated two major xanthomonadin esters from Xanthomonas juglandis (campestris); additional analysis showed that both esters were phospholipid-like compounds. The phospholipid-like structure was further supported by the findings that xanthomonadins are always coextracted from $X$. campestris pv. campestris together with phospholipids, such as phosphatidylcholine, phosphatidylethanolamine, and cardiolipin (Moser et al. 2014). Nevertheless, because of the difficulty in obtaining pure and stable pigments, the detailed chemical compositions and structures of xanthomonadins remain unclear. 
A 25.4-kb pig genomic region containing seven transcriptional units (pigA-G) was first identified to be responsible for xanthomonadin biosynthesis in $X$. campestris pv. campestris (Poplawsky and Chun 1997; Poplawsky et al. 1993). pig-like gene clusters were also reported in $X$. oryzae pv. oryzae and Lysobacter enzymogenes (Goel et al. 2002; Wang et al. 2013), and disruption of the genes for enzymes in the shikimate pathway abolished X. oryzae pv. oryzae xanthomonadin biosynthesis (Goel et al. 2001; Park et al. 2009). In the genome of X. campestris pv. campestris strain ATCC33913, the putative pig cluster is composed of the 20 genes Xcc3996 to Xcc4015. Pseudomonas aeruginosa strain M18 expressing this $X$. campestris pv. campestris pig gene cluster produced a darker yellow pigment that had structural changes, including the substitution of chlorine for bromine on the aryl ring (Cao et al. 2018). Single-gene deletion analysis showed that only 11 genes of the pig cluster (named $\left.x a n A_{2} B_{2} C D E F G H K L M\right)$ are essential for xanthomonadin biosynthesis in X. campestris pv. campestris (Cao et al. 2018). Among them, xanB2 (Xcc4014) was shown to encode a bifunctional chorismatase that hydrolyzes chorismate into 3-hydroxybenzoic acid (3-HBA) and 4-HBA in X. campestris pv. campestris and $X$. oryzae pv. oryzae (Zhou et al. 2013a, b). The gene $x a n A 2$ (Xcc4015) was found to encode an ATP-dependent 3-HBA:acyl carrier protein (ACP) ligase, whereas $x a n C$ (Xcc4012) encoded an ACP. Both XanA2 and XanC work together to activate 3-HBA to generate 3-HBA-S-ACP, which is needed for the initiation of xanthomonadin biosynthesis (Cao et al. 2018). The other proteins may be involved in xanthomonadin biosynthesis via an unusual type II polyketide synthase pathway (Cao et al. 2018). In our previous results, we showed that the proposed signal molecule DF, which was required for xanthomonadin production, was actually a mixture of 3-HBA and 4-HBA. Subsequently, it was proposed that 3-HBA and 4-HBA were intermediates in xanthomonadin and coenzyme $\mathrm{Q}(\mathrm{CoQ})$ biosynthesis, respectively, in both $X$. campestris pv. campestris and $X$. oryzae pv. oryzae (He et al. 2011; Zhou et al. 2013a). However, accumulating evidence suggests that 4-HBA might also be involved in xanthomonadin biosynthesis. First, exogenous addition of 4-HBA to the pigment-deficient mutant $\triangle x a n B 2$ resulted in the biosynthesis of darker yellow pigments (Zhou et al. 2013b). Second, our 2018 findings showed that XanA2, an ATPdependent 3-HBA:ACP ligase, can also activate 4-HBA in vitro (Cao et al. 2018). To further elucidate the roles of 3-HBA and 4-HBA in xanthomonadin biosynthesis, we applied three strategies to selectively eliminate 3-HBA, 4-HBA, or the production of both in the $X$. campestris pv. campestris wild-type strain XC1 (Fig. 1). Our results clearly demonstrated that $X$. campestris pv. campestris uses both 3-HBA and 4-HBA for xanthomonadin biosynthesis.

\section{MATERIALS AND METHODS}

Bacteria strains, plasmids, and growth condition. The bacterial strains and plasmids used in this study are listed in Supplementary Table S1. X. campestris pv. campestris strains were grown in nitrogen yeast glycerol (NYG) medium ( $5 \mathrm{~g} /$ liter of peptone, 3 $\mathrm{g} /$ liter of yeast extract, and $20 \mathrm{~g} /$ liter of glycerol, $\mathrm{pH}$ 7.0), nutrient agar (NA) medium ( $5 \mathrm{~g} /$ liter of peptone, $3 \mathrm{~g} /$ liter of beef extract, $10 \mathrm{~g} /$ liter of sucrose, and $1 \mathrm{~g} /$ liter of yeast extract, $\mathrm{pH}$ 7.0), or nutritionally poor XOLN medium $\left(0.7 \mathrm{~g} /\right.$ liter of $\mathrm{K}_{2} \mathrm{HPO}_{4}, 0.2 \mathrm{~g} /$ liter of $\mathrm{KH}_{2} \mathrm{PO}_{4}, 1.0$ g/liter of $\left(\mathrm{NH}_{4}\right)_{2} \mathrm{SO}_{4}, 0.1 \mathrm{~g} /$ liter of $\mathrm{MgCl}_{2} \cdot 6 \mathrm{H}_{2} \mathrm{O}, 0.01 \mathrm{~g} /$ liter of $\mathrm{FeSO}_{4} \cdot 7 \mathrm{H}_{2} \mathrm{O}, 0.001 \mathrm{~g} /$ liter of $\mathrm{MnCl}_{2} \cdot 4 \mathrm{H}_{2} \mathrm{O}, 0.0625 \%$ yeast extract, and $0.0625 \%$ peptone, $\mathrm{pH} 7.0$ ) at $28^{\circ} \mathrm{C}$. Escherichia coli strains were cultured at $37^{\circ} \mathrm{C}$ in Luria-Bertani (LB) medium ( $10 \mathrm{~g} /$ liter of tryptone, $5 \mathrm{~g} /$ liter of yeast extract, and $10 \mathrm{~g} /$ liter of $\mathrm{NaCl}, \mathrm{pH} 7.0$ ), and $P$. aeruginosa M18 and Klebsiella pneumoniae M5a1 were grown in LB medium at $28^{\circ} \mathrm{C}$. If necessary, antibiotics were added at the following concentrations: rifampicin (Rif), $25 \mu \mathrm{g} / \mathrm{ml}$; kanamycin $(\mathrm{Km}), 50 \mu \mathrm{g} / \mathrm{ml}$; spectinomycin, $50 \mu \mathrm{g} / \mathrm{ml}$; gentamycin $(\mathrm{Gm}), 20 \mu \mathrm{g} / \mathrm{ml}$ for $E$. coli and $30 \mu \mathrm{g} / \mathrm{ml}$ for $X$. campestris pv. campestris); and ampicillin, $100 \mu \mathrm{g} / \mathrm{ml}$.
Construction of gene or gene cluster overexpression in $X$. campestris pv. campestris. The coding regions of pchAB, $m h b D H I M$, and $p o b A$ were amplified by PCR using the primers in Supplementary Table S2. These genes were further cloned into the expression vector pBBR1MCS-2 (Km $\left.{ }^{\mathrm{R}}\right)$ (Kovach et al. 1995), and they were transferred to $X$. campestris pv. campestris strains by triparental mating. The positive transformants growing on NYG plate containing Rif $(25 \mu \mathrm{g} / \mathrm{ml})$ and $\mathrm{Km}(50 \mu \mathrm{g} / \mathrm{ml})$ were further confirmed by colony PCR. For single-copy $p \operatorname{ch} A B$ heterologous expression in $X$. campestris pv. campestris, the coding region of $p \operatorname{ch} A B$ plus the promoter region from pBBR-pch $A B$ was amplified and cloned into a versatile Mini-Tn7 delivery vector mini-Tn7T-Gm plasmid. The resultant construction was transformed into $X$. campestris pv. campestris by electroporation together with the helper plasmid pTNS2 (Choi and Schweizer 2006) (Supplementary Fig. S1). Chromosomal integration of mini-Tn7 was verified by PCR using three pairs of primers listed in Supplementary Figure S2.

Extraction and quantitative analyses of 3-HBA, 4-HBA, and salicylic acid. 3-HBA, 4-HBA, and salicylic acid (SA) extractions were performed as described (Zhou et al. 2013b) with minor modifications. Briefly, $2.5 \mathrm{ml}$ of supernatants of $X$. campestris pv. campestris culture were collected and adjusted to $\mathrm{pH} 4.0$ by addition of $1 \mathrm{M} \mathrm{HCl}$ prior to extraction with a twofold volume of ethyl acetate. The ethyl acetate fractions were then collected, and the solvent was removed by rotary evaporation to dryness at $40^{\circ} \mathrm{C}$. The residues were then dissolved in $0.1 \mathrm{ml}$ of methanol and submitted to high-performance liquid chromatography (HPLC) with a C18 reverse-phase column (Zorbax XDB; $5 \mu \mathrm{m}, 4.6 \times 150 \mathrm{~mm}$ ), which was eluted with methanol and water containing $0.05 \%$ formic acid in each phase $(25 / 75[\mathrm{vol} / \mathrm{vol}])$ at $1 \mathrm{ml} / \mathrm{min}$. Commercially available 3 HBA, 4-HBA, and SA were used as standards.

For liquid chromatography-mass spectrum (LC-MS) analysis of SA, the extracted sample or SA standard was injected into the ultraperformance liquid chromatography (UPLC) coupled with MS apparatus (Agilent UPLC1290-TOF-MS6230) under the following conditions: Agilent Zorbax XDB C18 reverse phase $(5 \mu \mathrm{m}, 4.6 \times$ $150 \mathrm{~mm}$ ) eluted with methanol and water containing $0.05 \%$ formic acid (25/75 [vol/vol]) at $0.4 \mathrm{ml} / \mathrm{min}$. The mass spectrum (MS) analysis was performed in the negative ion mode with an electrospray ionization source. Data were acquired in the centroid mode using Agilent MassHunter Workstation Data Acquisition Software (revision B.04).

Extraction and quantification of xanthomonadins. Pigments were extracted from $X$. campestris pv. campestris using previously described procedures (He et al. 2011). Briefly, $X$. campestris pv. campestris liquid cultures were grown until the stationary phase (optical density at $600 \mathrm{~nm}\left[\mathrm{OD}_{600}\right]=2.3$ ) in NYG medium. The cell pellets from $10 \mathrm{ml}$ of cultures were collected by centrifugation. Pigments were extracted with $1 \mathrm{ml}$ methanol by

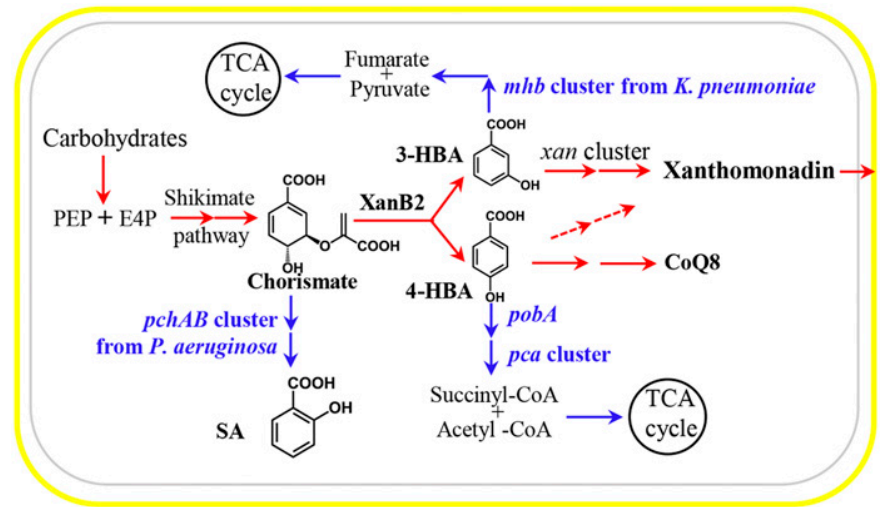

Fig. 1. The three strategies used to genetically interfere with xanthomonadin biosynthesis in this study. The solid lines indicate known pathways, and dotted red lines indicate postulated pathways in this study. CoQ, co-enzyme Q; E4P, erythrose-4-phosphate; HBA, hydroxybenzoic acid; PEP, phosphoenol-pyruvate; $\mathrm{SA}$, salicylic acid; TCA cycle, tricarboxylic acid cycle. 


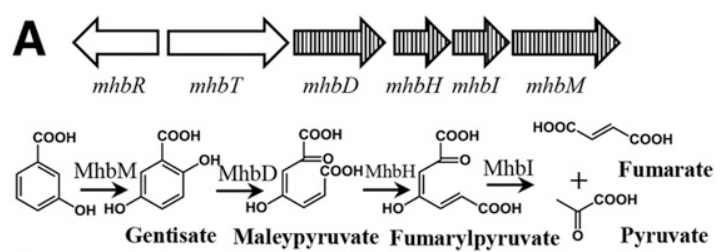

B
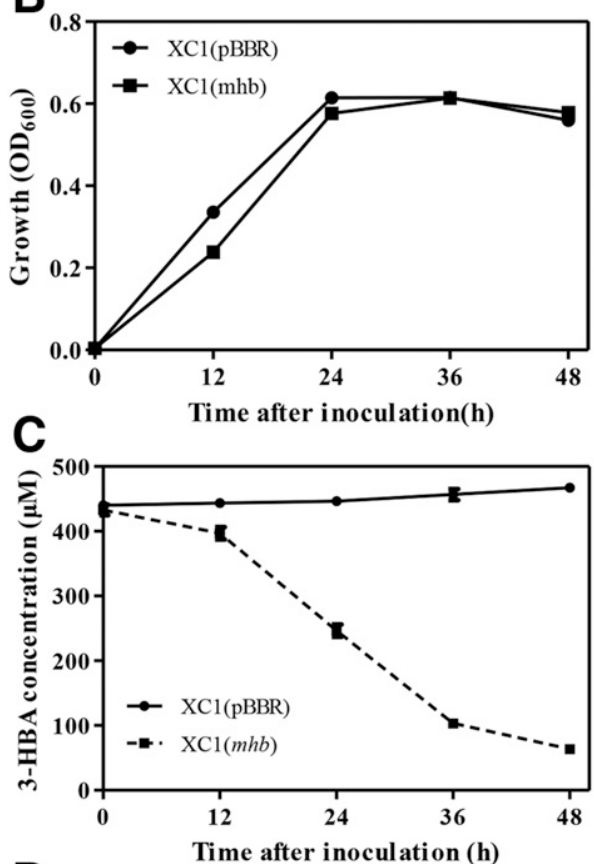

D
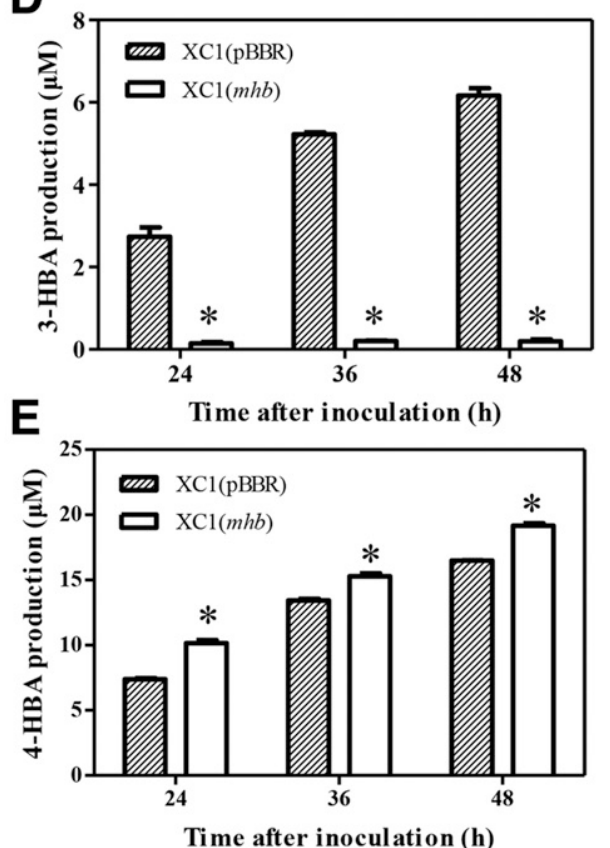

Fig. 2. The effects of heterologous expression of the 3-hydroxybenzoic acid (3-HBA) degradation gene cluster in Xanthomonas campestris pv. campestris. A, The $m h b$ gene cluster and putative 3-HBA degradation mechanism in Klebsiella pneumoniae. B, The time course of bacterial growth of X. campestris pv. campestris strains in nutritionally poor XOLN medium. C, The time course of 3-HBA degradation in XOLN medium. D, The 3-HBA levels of strains $\mathrm{XC1}(\mathrm{pBBR})$ and $\mathrm{XC1}(m h b)$ in nitrogen yeast glycerol $(\mathrm{NYG})$ medium. E, The 4-HBA levels of strains XC1(pBBR) and XC1 $(m h b)$ in NYG medium. Shown are averages for three biological replicates with standard deviation. $\mathrm{OD}$, optical density. ${ }^{*}$ Statistically significant differences with respect to strain $\mathrm{XC} 1(\mathrm{pBBR})(P \leq 0.05)$. shaking for $5 \mathrm{~min}$ at room temperature. The amount of pigment produced was expressed as the absorbance $\left(\mathrm{OD}_{441}\right)$ of crude pigment extracts as described previously (Poplawsky and Chun 1997). The methyl esters of xanthomonadins were prepared following a method previously described by Cao et al. (2018).

Exogenous addition of 3-HBA or 4-HBA. Exogenous addition of 3-HBA or 4-HBA (from Sigma; purity $>99 \%$ ) was performed following previously described methods (He et al. 2011). In liquid NYG culture, 3-HBA or 4-HBA was added to the $X$. campestris pv. campestris cultures when the $\mathrm{OD}_{600}$ reached 0.02 . Xanthomonadins were then extracted using the above-mentioned methods. $X$. campestris pv. campestris cultures were grown overnight on NYG agarose plates, diluted to an $\mathrm{OD}_{600}$ of 0.05 , and spotted at the agarose bars. Subsequently, either 3-HBA or 4-HBA was added to the upper end of the agarose bars. The resulting plates were sealed and incubated at $28^{\circ} \mathrm{C}$ for 2 days. The presence of yellow-colored colonies indicated the biosynthesis of xanthomonadins.

Quantitative determination of extracellular polysaccharide and ubiquinone. Extracellular polysaccharide (EPS) was extracted and quantified following methods previously described (He et al. 2011). The extraction and quantitative analysis of ubiquinone was performed as previously described with slight modifications (Zhou et al. 2013b); $20 \mathrm{ml}$ of $X$. campestris pv. campestris cultures were grown in NYG medium to an $\mathrm{OD}_{600}$ of 1.6 , and cells were then collected by centrifugation. After washing twice with $1 \times$ phosphate buffer saline (PBS), cell pellets were resuspended in $5 \mathrm{ml}$ of $0.2 \mathrm{M}$ acetate buffer $(\mathrm{pH}$ 5.6). Cryo-impacted cell homogenate was then subjected to sonication for a total of $90 \mathrm{~s}$. Hexane-acetone $(12.5 \mathrm{ml} ; 1: 1$ [vol/vol]) reaction mixture was added to the cell homogenate followed by further sonication and vortexing. The hexane fractions were collected, and the solvent was removed by rotary evaporation to dryness at $40^{\circ} \mathrm{C}$. The resulting residue was then dissolved in $1.5 \mathrm{ml}$ of chloroform-methanol $(1: 1[\mathrm{vol} / \mathrm{vol}])$ followed by washing with $0.75 \mathrm{ml}$ of $0.7 \% \mathrm{NaCl}$. The chloroform fraction was then collected, and the solvent was removed by rotary evaporation to dryness at $40^{\circ} \mathrm{C}$. The resulting residue was then dissolved in $100 \mu \mathrm{l}$ of methanol, and it was subjected to HPLC with a C18 reverse-phase column (Zorbax XDB; $5 \mu \mathrm{m}, 4.6 \times 150 \mathrm{~mm}$ ) eluting with methanol and isopropanol in each phase (50:50 [vol/vol]) for $20 \mathrm{~min}$ at a flow rate of $0.5 \mathrm{ml} / \mathrm{min}$ and ultraviolet detection at $275 \mathrm{~nm}$. Commercially available $\mathrm{CoQ}_{8}$ was used as a standard.

$X$. campestris pv. campestris virulence tests. $X$. campestris pv. campestris virulence in cabbage and radish was estimated by the leaf clipping method. Fresh cell cultures at an $\mathrm{OD}_{600}$ of 0.1 were used to inoculate clipped leaves of cabbage (cultivar Jingfeng no. 1) and Chinese radish (cultivar Manjianghong). The inoculated leaf was subsequently kept in an incubator at $28^{\circ} \mathrm{C}$ with $80 \%$ humidity, and lesion length was scored 10 days after inoculation. Fifteen leaves were inoculated with each tested strain. Each strain was tested in at least three separate experiments.

\section{RESULTS}

Heterologous expression of the $K$. pneumoniae 3-HBA degradation gene cluster in $X$. campestris pv. campestris results in the biosynthesis of a different xanthomonadin. Previous studies have proposed that the aryl ring moiety of xanthomonadin is derived from 3-HBA (He et al. 2011). To further confirm the role of 3 -HBA in xanthomonadin biosynthesis, the $m h b$ gene cluster ( $m h b D H I M)$, encoding the enzymes that cleave 3HBA into fumarate and pyruvate in K. pneumoniae (Xu et al. 2012), was cloned and overexpressed in the $X$. campestris pv. campestris wild-type strain $\mathrm{XC} 1$ via a constitutive promoter in a broad hostrange expression vector pBBR1MCS-2 (Fig. 2A). XC1 carrying the empty vector was used as a control. The resulting strains $\mathrm{XC} 1(m h b)$ and $\mathrm{XC} 1(\mathrm{pBBR})$ were grown in the following two media for functional analysis. First, when grown in the poor nutrient medium XOLN supplemented with $0.5 \mathrm{mM} 3$-HBA, no significant difference in bacterial growth was observed between the two strains 
(Fig. 2B). The levels of 3-HBA in cultures of strain $\mathrm{XC1}(\mathrm{mhb})$ decreased over time, whereas 3-HBA levels remained relatively stable in the control strain $\mathrm{XC1}$ (pBBR) cultures (Fig. 2C). Thus, the $m h b$ gene cluster from $K$. pneumoniae was functional in $X$. campestris pv. campestris. Second, although both strains produced 3-HBA, when cultured in glycerol-containing NYG medium in the absence of 3-HBA, the levels in the $\mathrm{XC1}(\mathrm{mhb})$ culture at 24,36 , and $48 \mathrm{~h}$ after inoculation were much lower than those in the $\mathrm{XC1}(\mathrm{pBBR})$ culture (Fig. 2D). Surprisingly, this overexpression of the $m h b$ cluster in $\mathrm{XC1}$ resulted in an increase of 4-HBA levels ranging from 14.2 to $18.8 \%$ depending on the growth stage (Fig. 2E).

We further investigated pigment biosynthesis in strains $\mathrm{XC1}$ $(\mathrm{pBBR})$ and $\mathrm{XC1}(m h b)$. On NYG agar, colonies of $\mathrm{XC1}(m h b)$ appeared darker than those of $\mathrm{XC1}(\mathrm{pBBR})$ (Fig. 3A); however quantitative, analysis $\left(\mathrm{OD}_{441}\right)$ revealed no significant difference in xanthomonadin yield in both strains (Fig. 3A). The absorption maximum for the $\mathrm{XC1}(\mathrm{pBBR})$-derived xanthomonadins occurred at approximately $441 \mathrm{~nm}$ (Fig. 3B). In contrast, the absorption maximum for the $\mathrm{XC} 1(\mathrm{mhb})$-derived dark yellow pigment occurred at approximately $430 \mathrm{~nm}$ (Fig. 3B). Exogenous addition of 3-HBA or 4-HBA to the $\mathrm{XC1}(\mathrm{pBBR})$ cultures had no significant effect on xanthomonadin biosynthesis (Supplementary Fig. S1), whereas the addition of 3 -HBA $(25 \mu \mathrm{M})$ to the $\mathrm{XC} 1(\mathrm{mhb})$ cultures restored bright yellow pigment biosynthesis and significantly increased pigment yield by $24 \%$ (Fig. 3A). Exogenous addition of 4-HBA $(25 \mu \mathrm{M})$ to $\mathrm{XC1}(\mathrm{mhb})$ cultures resulted in dark yellow pigment biosynthesis and an increased pigment yield of about 27\% (Fig. 3A). These findings suggest that, in absence of 3-HBA, 4-HBA is used for biosynthesis of the dark yellow pigment in the $\mathrm{XC1}(m h b)$ strain.

We have developed an ultrahigh performance liquid chromatographytime of flight mass spectrometry (UPLC-TOF-MS)-based method to characterize the methyl ester of the yellow pigments produced by strain XC1 (Cao et al. 2018). The main methyl ester of the $\mathrm{XC1}(\mathrm{pBBR})$-derived xanthomonadins was dibrominated with a putative formula of $\mathrm{C}_{24} \mathrm{H}_{22} \mathrm{Br}_{2} \mathrm{O}_{3}$ denoted Xanthomonadin-A (Fig. $3 \mathrm{C})$. However, the level of this pigment in strain $\mathrm{XC1}(\mathrm{mhb})$ was only $16 \%$ of that seen in the strain $\mathrm{XC1}(\mathrm{pBBR}$ ) (Fig. 3C), and the predominant pigment was a new methyl ester tentatively denoted Xanthomonadin-B (Fig. 3C). Xanthomonadin-B is structurally different from the previously characterized dibrominated Xanthomonadin-A.

Overexpression of the 4-HBA monooxygenase gene pobA significantly reduced xanthomonadin biosynthesis in $X$. campestris pv. campestris. A gene cluster required for 4HBA degradation was previously identified in $X$. campestris pv. campestris (Wang et al. 2015). One of the genes, pobA, encodes a 3-monooxygenase, which catalyzes the degradation of 4-HBA into protocatechuate prior to further aromatic ring cleavage (Fig. 4A). To investigate whether 4-HBA is involved in xanthomonadin biosynthesis, we generated the $p o b A$ deletion and overexpression strains $\mathrm{XC1}(p o b A), \triangle p o b A(\mathrm{pBBR})$, and $\triangle p o b A(p o b A)$ and measured their biosynthesis of 3-HBA, 4-HBA, and xanthomonadins. When grown in NYG liquid medium, all four strains showed similar growth rates (Fig. 4B). Deletion of pobA in strain XC1 had little effect on the levels of 3-HBA and 4-HBA (Fig. 4C and D). Overexpression of pobA in strains $\mathrm{XC1}$ or $\triangle p o b A$ resulted in a reduction of 4-HBA levels ranging from 88 to $98 \%$; however, 3HBA levels showed significant increases ranging from 18 to $23 \%$ at various times after inoculation (Fig. $4 \mathrm{C}$ and D).

When grown on NYG agar, the colonies of strains $\mathrm{XC1}$ (pobA) and $\triangle p o b A(p o b A)$ appeared lighter yellow in color than strains $\mathrm{XC1}(\mathrm{pBBR})$ and $\triangle p o b A(\mathrm{pBBR})$ (Fig. 4E). Quantitative analysis showed that deletion of $p o b A$ had no significant effect on pigment yield; however, overexpression of $p o b A$ in $\mathrm{XC} 1$ or $\triangle p o b A$ led to 23 and $25 \%$ decreases in pigment yield, respectively (Fig. 4E). The absorption maximum analysis and UPLC-TOF-MS analysis indicated that the predominant $\mathrm{XC1}$ (pobA)-derived xanthomonadin was Xanthomonadin-A (Fig. 3B and C). Quantitative analysis of
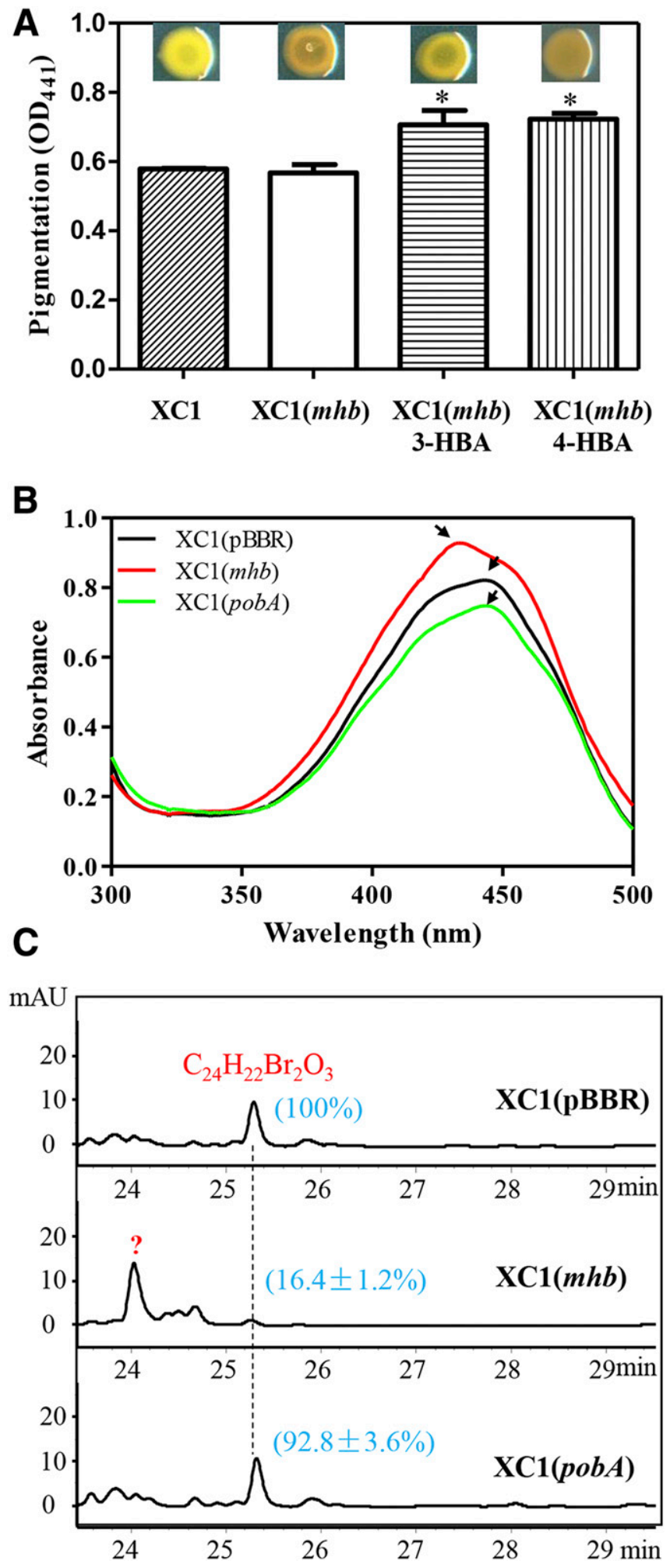

Fig. 3. The effect of heterologously expressing the $m h b$ gene cluster on xanthomonadin biosynthesis in Xanthomonas campestris pv. campestris. A, Colony pigmentation and xanthomonadin biosynthesis in X. campestris pv. campestris strains. Shown are averages for three biological replicates with standard deviation. HBA, hydroxybenzoic acid; OD, optical density. *Statistically significant differences with respect to strain $\mathrm{XC1}(m h b)(P \leq 0.05)$. B, Absorption spectra of crude xanthomonadin extracts from $\mathrm{XC1}(\mathrm{pBBR})$, $\mathrm{XC1}(\mathrm{mhb})$, and pobA expression strain $\mathrm{XC1}(\mathrm{pobA})$. The absorption maxima are indicated with arrows. C, High-performance liquid chromatography analysis of the methyl esters in strains $\mathrm{XC1}(\mathrm{pBBR}), \mathrm{XC1}(m h b)$, and $\mathrm{XC1}(p o b A)$. The dibrominated xanthomonadin methyl ester is indicated by a dashed line, and their relative ratios are indicated by percentages in parentheses. 

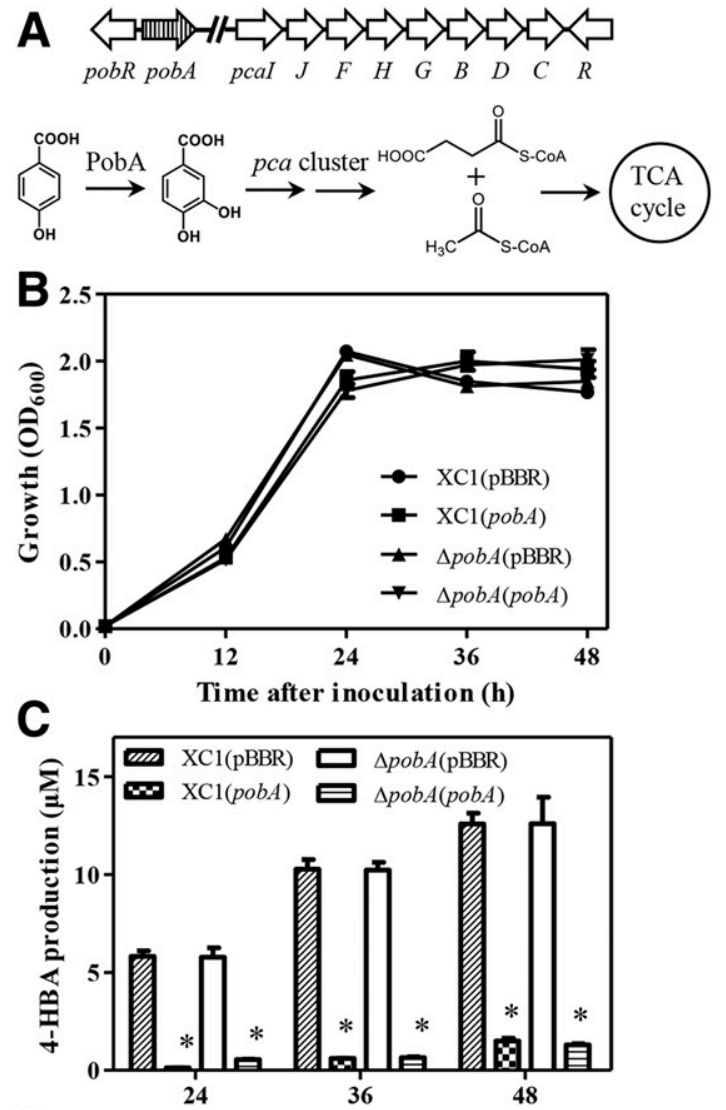

D Time after inoculation (h)
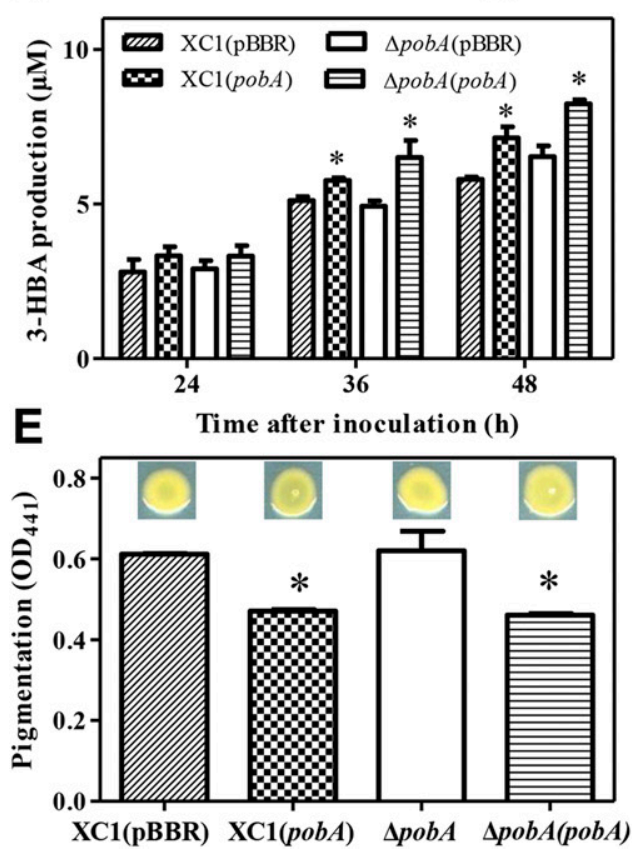

Fig. 4. Overexpression of $p o b A$ significantly reduced xanthomonadin biosynthesis in Xanthomonas campestris pv. campestris. A, The gene cluster for 4-hydroxybenzoic acid (4-HBA) degradation and the proposed degradation pathway in X. campestris pv. campestris. TCA cycle, tricarboxylic acid cycle. B, Time course of bacterial growth in Nitrogen yeast glycerol medium. C, The 4-HBA biosynthesis in the pobA deletion and overexpression strains $\triangle p o b A(\mathrm{pBBR}), \mathrm{XC1}(p o b A)$, and $\triangle p o b A(p o b A)$. D, The 3-HBA biosynthesis in the pobA deletion and overexpression strains $\triangle p o b A(\mathrm{pBBR}), \mathrm{XC1}(p o b A)$, and $\triangle p o b A(p o b A)$. E, Colony pigmentation and xanthomonadin biosynthesis in pobA deletion and overexpression strains $\triangle p o b A(\mathrm{pBBR}), \mathrm{XC1}(p o b A)$, and $\triangle p o b A(p o b A)$. Shown are averages for three biological replicates with standard deviation. OD, optical density. *Statistically significant differences with respect to strain $\mathrm{XC1}(\mathrm{pBBR})(P \leq 0.05)$. methyl esters showed that Xanthomonadin-A produced in the pobA overexpression strain $\mathrm{XC1}(p o b A)$ was about $8 \%$ lower than that in the wild-type strain $\mathrm{XC1}$ (pBBR) (Fig. 3C).

The effect of the $p c h A B$ cluster on xanthomonadin biosynthesis depends on its copy number. The $p c h A-G$ cluster is involved in the biosynthesis of the siderophore pyochelin in P. aeruginosa (Gaille et al. 2002, 2003). pchA encodes an isochorismate synthase, and $p c h B$ encodes an isochorismate pyruvate-lyase, which hydrolyzes chorismate into SA via a twostep reaction (Supplementary Fig. S2A). Our previous results showed that chorismate is the substrate of a bifunctional chorismatase XanB2, which produces both 3-HBA and 4-HBA; 3 -HBA is subsequently used as a precursor for xanthomonadin biosynthesis in $X$. campestris pv. campestris and $X$. oryzae $\mathrm{pv}$. oryzae (Zhou et al. 2013a, b). To further verify the roles of 3-HBA and 4-HBA in xanthomonadin biosynthesis, the $p c h A B$ gene cluster from $P$. aeruginosa strain M18 was expressed in strain XC1 in either of two ways: (i) via the multicopy expression vector pBBR1MCS-2 $\left(\mathrm{Kan}^{\mathrm{R}}\right)[\mathrm{XC1}(p c h A B)]$ or (ii) via the vector mini-Tn7T-Gm, which integrates a single copy of the $p \operatorname{ch} A B$ gene cluster into the chromosome (XC1::pchAB). We hypothesized that the introduced SA biosynthetic pathway would compete with the XanB2dependent xanthomonadin biosynthetic pathway for the common precursor, chorismate.

Using UPLC-TOF-MS analysis as described by Wang et al. (2015), SA was detected in $\mathrm{XC1}(p c h A B)$ but not $\mathrm{XC1}(\mathrm{pBBR})$ cultures (Supplementary Fig. S2B and C). The SA levels in $\mathrm{XC1}(p \operatorname{ch} A B)$ cultures increased over time, with the highest level of 292.3 $\mu \mathrm{M}$ observed at $48 \mathrm{~h}$ after inoculation (Fig. 5A). This overexpression of $p c h A B$ in XC1 grown in $\mathrm{NYG}$ medium significantly reduced bacterial growth and the biosynthesis of 3HBA, 4-HBA, and xanthomonadins (Fig. 5A to C). The subsequent exogenous addition of 3 -HBA $(25 \mu \mathrm{M})$ or 4 -HBA $(25 \mu \mathrm{M})$ to the $\mathrm{XC1}(p c h A B)$ cultures resulted in bright or dark yellow pigment biosynthesis, respectively (Fig. 5C). Further analysis revealed two pigment absorption maxima at approximately 420 and approximately $450 \mathrm{~nm}$ with $\mathrm{XC1}(p c h A B)$ in the presence of 3-HBA, and in the presence of 4-HBA, a pigment absorption maximum at approximately $430 \mathrm{~nm}$ was observed, similar to what was observed with strain $\mathrm{XC1}(\mathrm{mhb})$ (Fig. 5D).

Next, to avoid the depression of $\mathrm{XC} 1$ growth owing to the high copy number of plasmid-borne $p \operatorname{ch} A B$, a single copy of the $p \operatorname{ch} A B$ cluster was integrated into the $X$. campestris pv. campestris chromosome at the att $\operatorname{Tn} 7$ site using the mini-Tn7T-Gm vector (Choi and Schweizer 2006) (Supplementary Fig. S3). The resulting strain $\mathrm{XC1}:: p c h A B$ showed a growth pattern similar to the wild-type strain XC1 in NYG medium (Fig. 6A). It produced less SA than the $p c h A B$ overexpression strain $\mathrm{XC1}(p \operatorname{ch} A B)$, with a maximum SA level of about $120 \mu \mathrm{M}$ observed at $48 \mathrm{~h}$ after inoculation (Fig. 6A). The levels of 3-HBA and 4-HBA in the XC1::pchAB cultures were 2.5 and $4.7 \mu \mathrm{M}$, respectively, which were 35 and $41 \%$, respectively, of those observed in the XC1 culture at $48 \mathrm{~h}$ after inoculation (Fig. $6 \mathrm{~B})$. When grown on NYG agar, the colonies of strains $\mathrm{XC} 1$ and

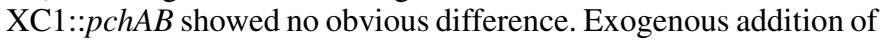
3-HBA had no effect on colonies color of strain XC1::pchAB, whereas the addition of 4-HBA in $\mathrm{XC} 1:: p \operatorname{ch} A B$ resulted in biosynthesis of dark yellow pigmentation. Further quantitative analysis showed that xanthomonadin production in strain $\mathrm{XC} 1$ :: pch $A B$ was $90.0 \%$ of that observed with strain $\mathrm{XC1}$ as judged by $\mathrm{OD}_{441}$ values of pigment extracts (Fig. 6C). The addition of 3-HBA or 4-HBA at a final concentration of $25 \mu \mathrm{M}$ to the $\mathrm{XC} 1:: p c h A B$ culture restored pigment production to the level of $\mathrm{XC1}$ (Fig. 6C).

To further rule out the possibility that the $p c h A B$-dependent SA might be involved in or interfere with pigment biosynthesis, SA was exogenously added to XC1 cultures at final concentrations of 0.25 and $0.50 \mathrm{mM}$, respectively. SA had little effect on the biosynthesis of 3-HBA, 4-HBA, or xanthomonadins at $48 \mathrm{~h}$ after inoculation (Supplementary Fig. S4). 

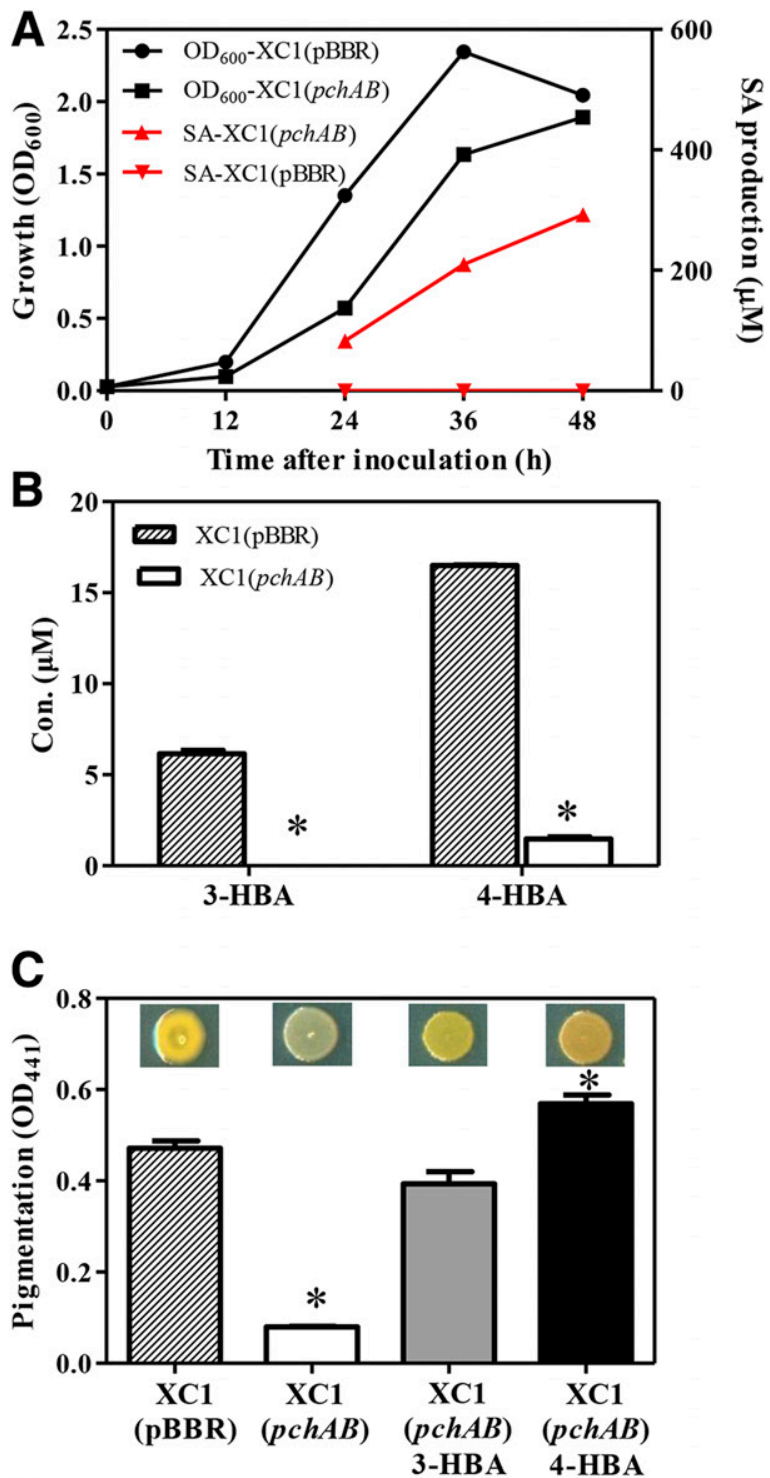

D

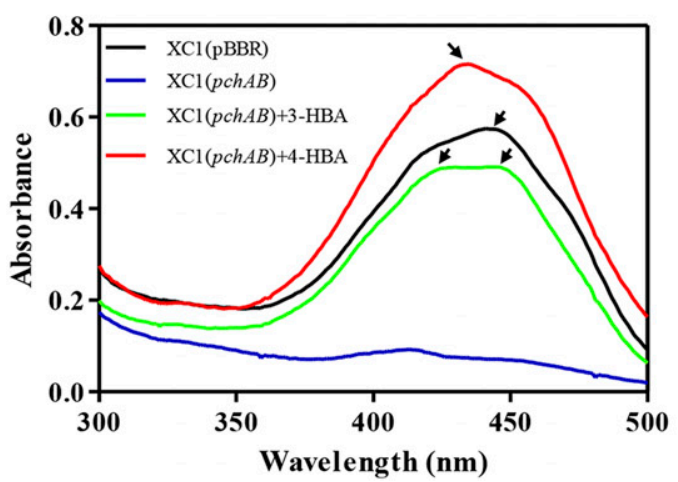

Fig. 5. The effect of $p c h A B$ overexpression on bacterial growth and 3hydroxybenzoic acid (3-HBA), 4-HBA, and xanthomonadin biosynthesis in Xanthomonas campestris pv. campestris. A, The time course of bacterial growth (black curves) and salicylic acid (SA) biosynthesis (gray/red curve) in NYG (nitrogen yeast glycerol) medium. B, The 3-HBA and 4-HBA levels in $\mathrm{XCl}(\mathrm{pBBR})$ and $\mathrm{XC1}(p c h A B)$ cultures $48 \mathrm{~h}$ after inoculation. $\mathrm{C}$, Colony pigmentation and xanthomonadin biosynthesis in strains $\mathrm{XCl}(\mathrm{pBBR})$ and $\mathrm{XCl}(p c h A B)$. OD, optical density. *Statistically significant differences with respect to the strain XC1(pBBR) $(P \leq$ 0.05). D, Absorption spectra of crude xanthomonadin extracts from strains $\mathrm{XC1}(\mathrm{pBBR})$ and $\mathrm{XC1}(p c h A B)$ in the presence of 3-HBA or 4-HBA. The absorption maxima are indicated with arrows. Combined with Figure $3 \mathrm{~B}$, the absorption maxima of crude xanthomonadin extracts from strains $\mathrm{XC1}(\mathrm{pBBR}), \mathrm{XC1}(m h b)$, and $\mathrm{XC1}(p o b A)$ were 441,430 , and $441 \mathrm{~nm}$, respectively, whereas the $\mathrm{XC1}(p c h A B)$ strain had no obvious absorption maxima. Shown are averages for biological replicates repeats with standard deviation.
Interference with 3-HBA and/or 4-HBA biosynthesis also affected a range of other phenotypes in $X$. campestris pv. campestris. Previous findings showed an extensive cross-talk between the xanthomonadin biosynthetic pathway and those of other secondary metabolites, such as EPS and diffusible signal factor (DSF) family signals and CoQ8 (Cao et al. 2018; Zhou et al. 2013b). Thus, we further analyzed the biosynthesis of extracellular enzymes EPS and CoQ8 in the above-mentioned strains, which were compromised for the production of 3-HBA, 4-HBA, or both. HPLC analysis of CoQ8 extracts showed that overexpression of

A

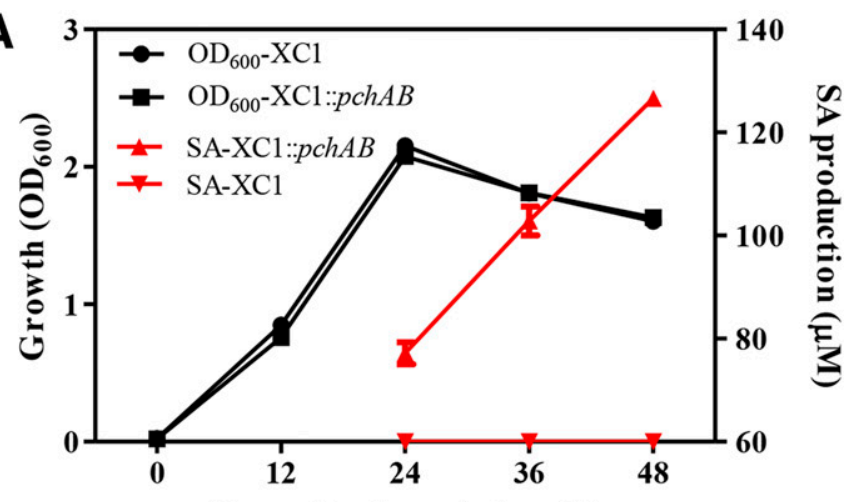

Time after inoculation (h)
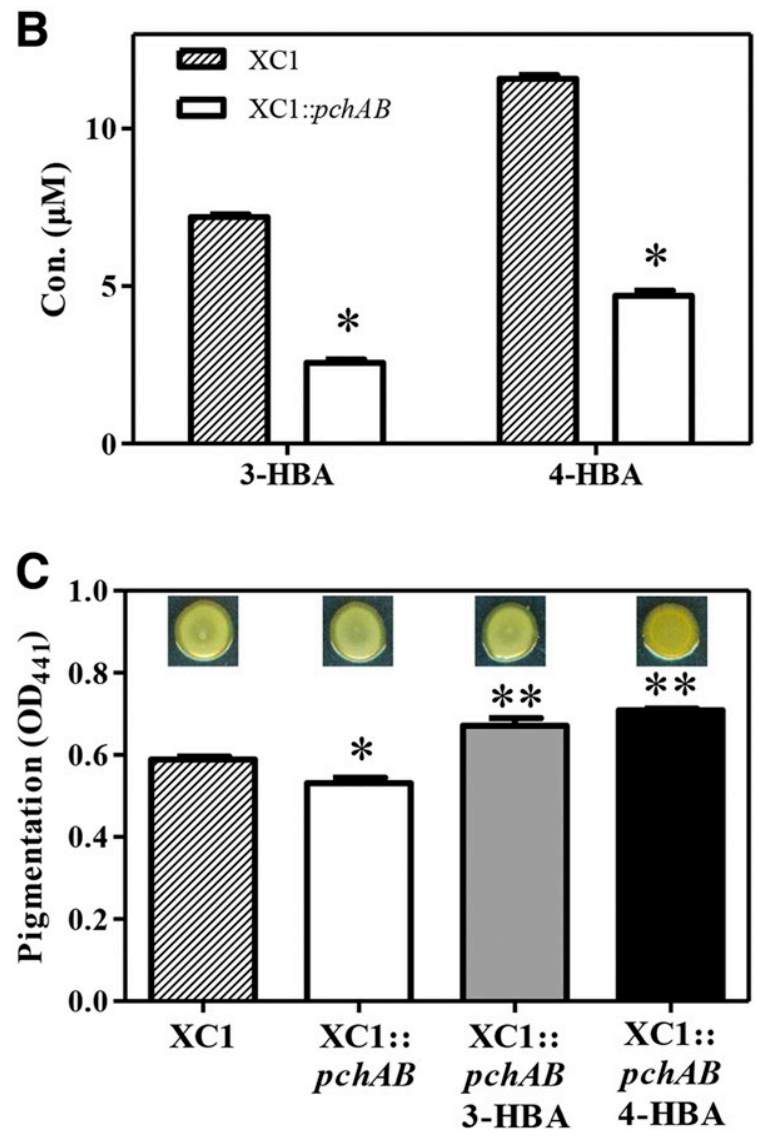

Fig. 6. The effect of a single copy of the $p c h A B$ cluster on bacterial growth and 3hydroxybenzoic acid (3-HBA), 4-HBA, and xanthomonadin biosynthesis in Xanthomonas campestris pv. campestris. A, Time course of bacterial growth (black curves) and salicylic acid (SA) biosynthesis (gray/red curve) in NYG (nitrogen yeast glycerol) medium. B, The 3-HBA and 4-HBA levels in $\mathrm{XC1}$ and $\mathrm{XC1}:: p c h A B$ cultures $48 \mathrm{~h}$ after inoculation. $\mathbf{C}$, Colony pigmentation and xanthomonadin biosynthesis in strains $\mathrm{XC} 1$ and $\mathrm{XC} 1:: p c h A B$. Shown are averages for three biological replicates with standard deviation. OD, optical density. *Statistically significant differences with respect to strain XC1 $(P \leq 0.05)$. **Statistically significant differences with respect to strain $\mathrm{XCl}(\mathrm{pBBR})(P \leq 0.01)$. 
$p c h A B$ or $p o b A$ in $\mathrm{XC} 1$ significantly reduced $\mathrm{CoQ} 8$ biosynthesis (Fig. 7A). CoQ8 levels in strains $\mathrm{XC1}(p c h A B)$ and $\mathrm{XC1}$ (pobA) were 69 and 26\%, respectively, of those observed in XC1(pBBR). Heterologous expression of the $m h b$ gene cluster had no effect on CoQ8 biosynthesis (Fig. 7A). The overexpression of the pchAB gene cluster or $p o b A$ also significantly reduced EPS biosynthesis (Fig. 7B). The EPS levels (early stationary phase, $\mathrm{OD}_{600}=2.3$ ) in cultures of $\mathrm{XC} 1(p c h A B)$ and $\mathrm{XC1}(p o b A)$ were 0.6 and $1.2 \mathrm{mg} / \mathrm{ml}$, respectively. These were much lower than the $2.0 \mathrm{mg} / \mathrm{ml}$ observed in the wild-type culture (Fig. 7B). As with CoQ8, the heterologous expression of the $m h b$ gene cluster had no effect on EPS biosynthesis.

Interference with 3-HBA and/or 4-HBA biosynthesis in $X$. campestris pv. campestris led to reduced virulence. Xanthomonadins, EPS, CoQ8, and extracellular enzymes are important factors needed for $X$. campestris pv. campestris adaptation to different host plant ecological niches and virulence (Büttner and Bonas 2010). In this study, we further evaluated the virulence of four $X$. campestris pv. campestris strains, XC1(pBBR), $\mathrm{XC1}(m h b), \mathrm{XC1}(p o b A)$, and $\mathrm{XC1}(p c h A B)$, by measuring lesion lengths on the leaves of cabbage and Chinese radish at 10 days postinoculation. Overexpression of the $m h b$ gene cluster or pobA led to similar reductions in virulence on cabbage and radish, with average lesion length reduced by 18 to 23 and 22 to $30 \%$,
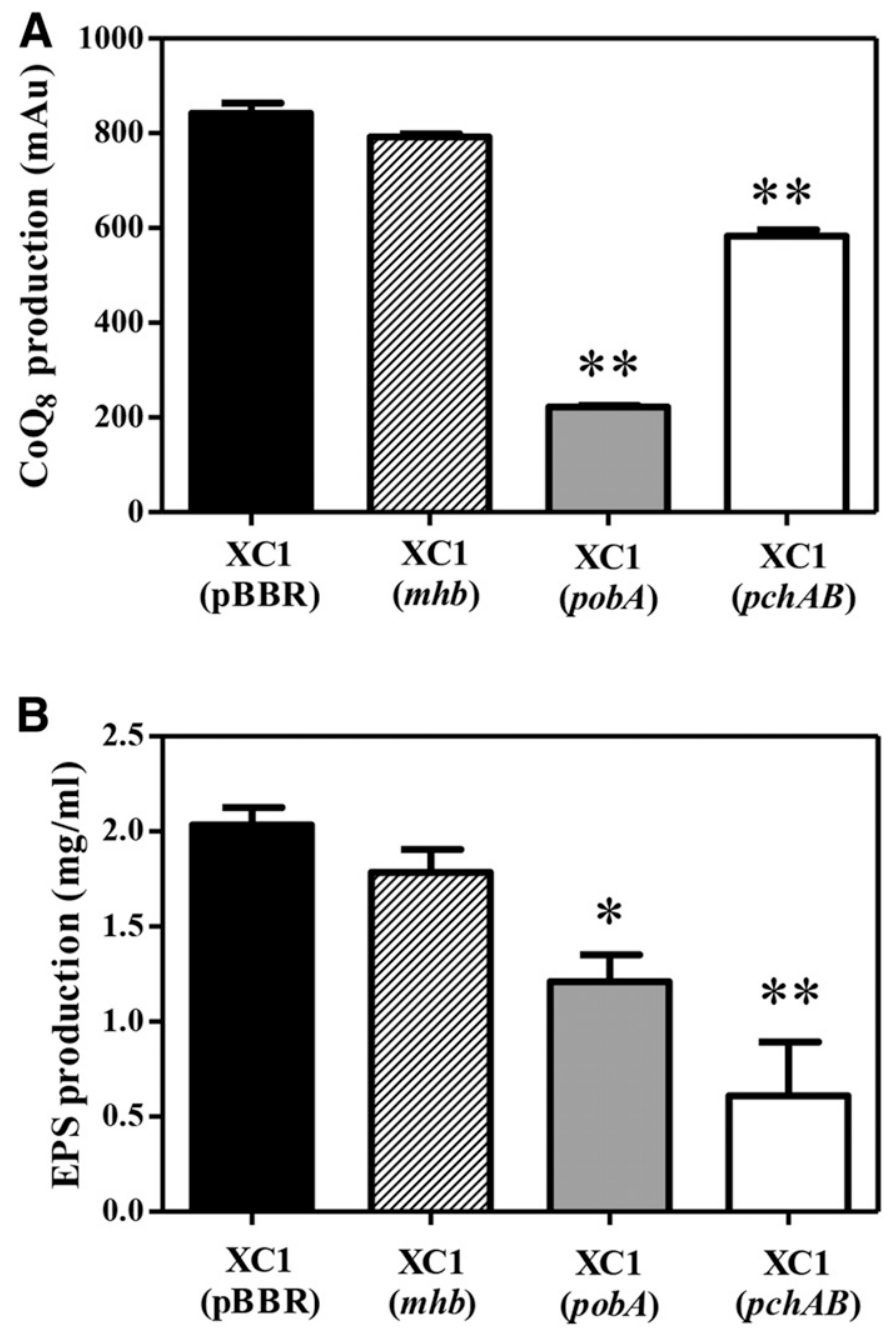

Fig. 7. Overexpression of $m h b$, pobA, and $p c h A B$ affected pleiotropic phenotypes in Xanthomonas campestris pv. campestris. A, Coenzyme Q8 (CoQ8) biosynthesis. B, Extracellular polysaccharide biosynthesis. EPS, extracellular polysaccharide. *Statistically significant differences with respect to strain $\mathrm{XC1}$ (pBBR) $(P \leq 0.05)$; **statistically significant differences with respect to strain $\mathrm{XC1}(\mathrm{pBBR})(P \leq 0.01)$. respectively (Fig. 8). However, overexpression of the $p c h A B$ gene cluster led to a more substantial reduction of pathogenicity, with the average lesion length reduced by $71 \%$ on cabbage and $77 \%$ on Chinese radish (Fig. 8). This large reduction in pathogenicity may in part have been because of the compromised growth effect of the $p c h A B$ gene cluster as well as the production of SA and its effect on host resistance induction.

\section{DISCUSSION}

XanB2, a new bifunctional chorismatase responsible for 3-HBA and 4-HBA biosynthesis from the common precursor chorismite, is

A

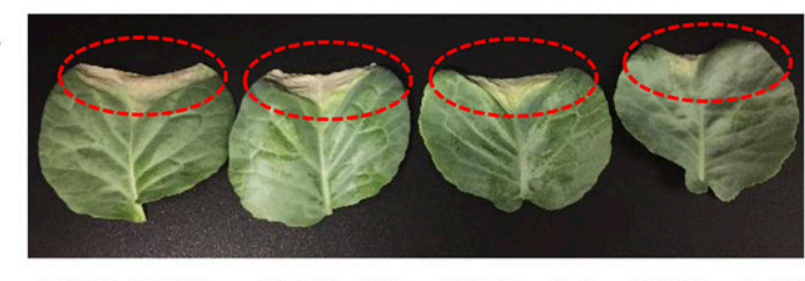

$\mathrm{XCl}(\mathrm{pBBR}) \quad \mathrm{XCl}(m h b) \quad \mathrm{XCl}(p o b A) \quad \mathrm{XCl}(p c h A B)$

B

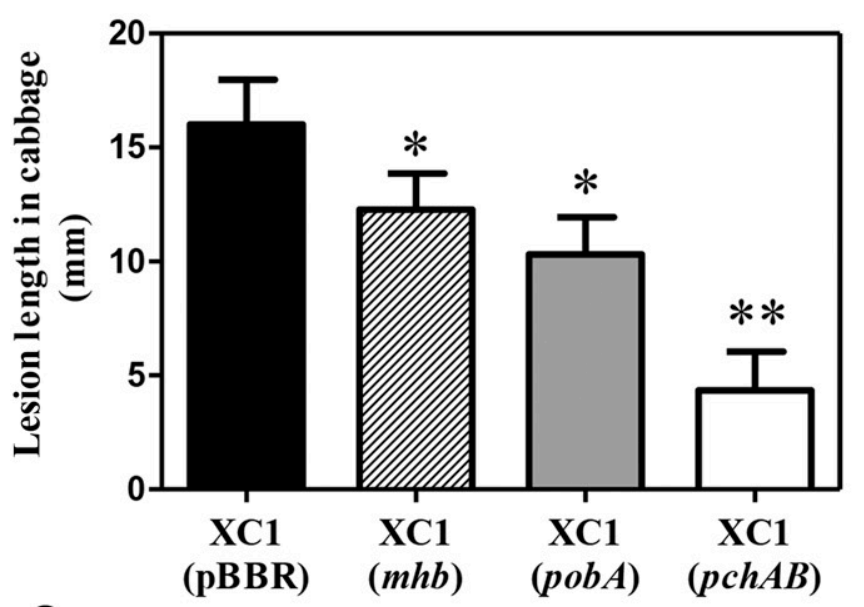

C

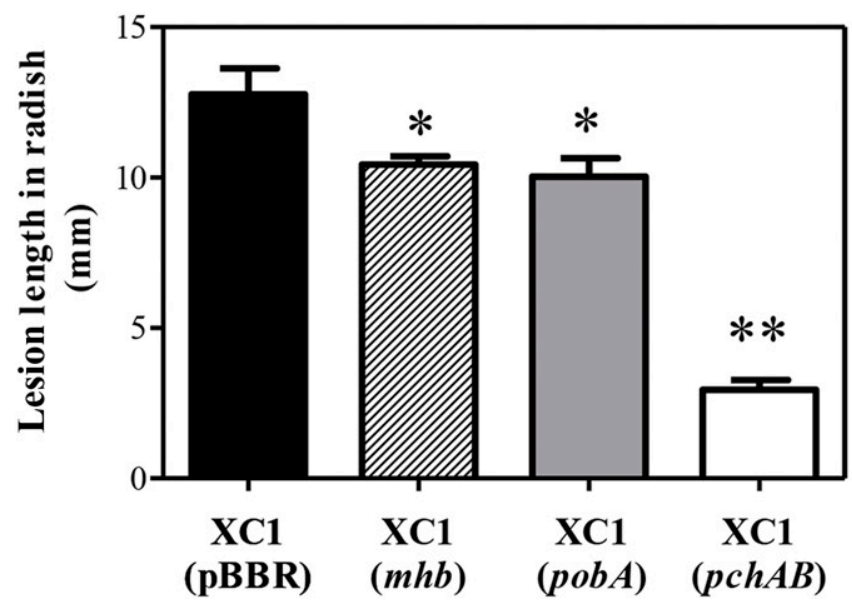

Fig. 8. Overexpression of $m h b$, pobA, and $p c h A B$ impaired Xanthomonas campestris pv. campestris pathogenicity. A, Infected cabbage leaves. From left to right: leaves infected by $\mathrm{XC} 1(\mathrm{pBBR}), \mathrm{XC1}(m h b), \mathrm{XC} 1(p o b A)$, and $\mathrm{XC1}(p \operatorname{ch} A B)$. The infection phenotypes on cabbage are labeled with red dotted ellipses. B, Quantitative analysis of the lesion length in infected cabbage leaves. C, Quantitative analysis of the lesion length in infected radish leaves. Shown are averages for 15 leaves with standard deviation. *Statistically significant differences with respect to strain $\mathrm{XC1}(\mathrm{pBBR})(P \leq 0.05)$; **statistically significant differences with respect to strain $\mathrm{XC1}(\mathrm{pBBR})(P \leq 0.01)$. 
essential for xanthomonadin biosynthesis in $X$. campestris $\mathrm{pv}$. campestris (Zhou et al. 2013a, b), and the exogenous addition of either 3-HBA or 4-HBA to strain $\triangle x a n B 2$ differentially restored pigment production (He et al. 2011). Thus, analysis of the $x a n B 2$ deletion mutant could not differentiate the possibly unique roles of 3-HBA and 4-HBA in the biosynthesis of xanthomonadins or the expression of other phenotypes, and an alternative strategy was required. In this study, we used genetic approaches to selectively eliminate 3-HBA, 4-HBA, or the production of both in $X$. campestris pv. campestris. The resulting strains were shown to be different in pigmentation, virulence factor production, and virulence. These findings suggest that both 3 -HBA and 4-HBA are used in xanthomonadin production in X. campestris pv. campestris. When both 3-HBA and 4-HBA are present, X. campestris pv. campestris prefers 3-HBA for xanthomonadin biosynthesis. If 3HBA is not present, then 4-HBA is used for a structurally uncharacterized xanthomonadin.

Previously, results showed that XanB2 acts on chorismate to produce similar levels of 3-HBA and 4-HBA in vitro (Zhou et al. 2013b). HPLC analysis also detected similar levels of exogenous 3HBA and 4-HBA in either X. campestris pv. campestris or X. oryzae pv. oryzae cultures (Zhou et al. 2013a, b). Findings in 2018 showed that XanA2, a key enzyme needed for the activation of 3-HBA during the initiation of xanthomonadin biosynthesis, had an even higher activity toward 4-HBA than 3-HBA in vitro (Cao et al. 2018). These findings suggest that the $X$. campestris pv. campestris wildtype strain should produce similar amounts of 3-HBA-derived Xanthomonadin-A and 4-HBA-derived Xanthomonadin-B. However, this study clearly shows that the production of the 3-HBAderived Xanthomonadin-A is dominant over the production of the 4-HBA-derived Xanthomonadin-B with the wild-type strain XC1 in vitro (Figs. 3A and 4E). The explanation for this contradiction may be that there are at least two 4-HBA-utilizing pathways in $X$. campestris pv. campestris: the UbiA-dependent CoQ biosynthetic pathway and the XanA2-dependent xanthomonadin biosynthetic pathway (Zhou et al. 2013b, 2019). Both pathways compete for the precursor 4-HBA. Intracellularly, UbiA may have a higher affinity toward 4-HBA than XanA2 so that most 4-HBA effluxes into the CoQ8 biosynthetic pathway. Assays to determine the affinities of XanA2 and UbiA toward 4-HBA will help to elucidate this possibility in the future.

$\mathrm{SA}$, an analog of 3-HBA and 4-HBA, is an important plant hormone that regulates plant immune responses in response to pathogen infection (Pieterse et al. 2012; Yuan et al. 2007). Our previous study showed that $X$. campestris pv. campestris could not use SA for xanthomonadin biosynthesis (He et al. 2011). This study further shows that the exogenous addition of SA has no effect on xanthomonadin biosynthesis (Supplementary Fig. S3). This is consistent with the finding that XanA2 could not activate SA to form salicyl-ACP (Cao et al. 2018). However, this study also shows that the overexpression of pchAB in $X$. campestris pv. campestris results in endogenous SA biosynthesis and a loss of xanthomonadin biosynthesis (Fig. 6C). This does not mean that SA inhibits xanthomonadin biosynthesis. Overexpression of $p \operatorname{ch} A B$ led to the excessive use of chorismate, which is an important biochemical intermediate for the synthesis of many alkaloids and other aromatic metabolites, including 3-HBA and 4-HBA (Zhou et al. 2013b). This indirectly led to limiting growth and xanthomonadin production in the strain $\mathrm{XC1}(p c h A B)$. Taken together, this study further confirms that the plant defense signal SA is not involved in xanthomonadin biosynthesis and also, cannot interfere with $X$. campestris pv. campestris xanthomonadin biosynthesis.

All characterized $X$. campestris pv. campestris xanthomonadins were derived from cells that were cultured in vitro. Although detailed xanthomonadin chemical structures are still not fully elucidated, the aryl-polyene ring is derived from 3-HBA (Andrewes et al. 1973, 1976). This study shows that Xanthomonadin-A, which was identified in both the wild-type strain and strain $\mathrm{XC} 1(p c h A B)$ in the presence of 3-HBA, corresponds to the known dibrominated xanthomonadins (Cao et al. 2018) (Figs. 3C and 5D). However, Xanthomonadin-B, which was produced by $\mathrm{XC1}(m h b)$ strain and strain $\mathrm{XC} 1(p c h A B)$ in the presence of 4-HBA, seems to be a new xanthomonadin variant (Figs. $3 \mathrm{C}$ and 5D). The detailed chemical structure and biological function of Xanthomonadin-B deserve further investigation.

Furthermore, as a phytopathogen, $X$. campestris pv. campestris has at least two very unique lifecycle stages in vivo. Initially, it lands on the host plant leaf surface, initiates an epiphytic stage, and increases its numbers. Subsequently, under favorable conditions, it may travel to the hydathodes, enter the plant, and initiate its pathogenic stage (Hugouvieux et al. 1998). Previous studies indicated that xanthomonadins may be needed by the pathogen in vivo for both the epiphytic and pathogenic stages (Poplawsky et al. 2000); however, it is not known which of the xanthomonadin variants are produced at either stage. Thus, the biological significance of these finding that $X$. campestris pv. campestris produces different xanthomonadins in response to the availability of 3-HBA, 4-HBA, or both deserves further investigation. The pathogen may selectively use either 3-HBA or 4-HBA in vivo to emphasize the production of the xanthomonadin variant most suited to its current life stage. We also observed that 3-HBA and 4-HBA had differential effects on the production of CoQ8 and EPS; 4-HBA seemed to have a role in promoting their production, whereas 3HBA did not. This observation is intriguing and warrants further investigation.

\section{ACKNOWLEDGMENTS}

We thank Ningyi Zhou for providing strain Klebsiella pneumoniae M5a1.

\section{LITERATURE CITED}

Andrewes, A. G., Hertzberg, S., Liaaen-Jensen, S., and Starr, M. P. 1973. The Xanthomonas "Carotenoids"-non-carotenoid brominated aryl-polyene esters. Acta Chem. Scand. 27:2383-2395.

Andrewes, A. G., Jenkins, C. L., Starr, M. P., Shepherd, J., and Hope, H. 1976. Structure of xanthomonadin I, a novel dibrominated aryl-polyene pigment produced by the bacterium Xanthomonas juglandis. Tetrahedron Lett. 17: 4023-4024.

Aririatu, L. E., and Kester, A. S. 1985. Isolation and characterization of the pigment esters of Xanthomonas juglandis (campestris). Microbiology 131: 2047-2052.

Büttner, D., and Bonas, U. 2010. Regulation and secretion of Xanthomonas virulence factors. FEMS Microbiol. Rev. 34:107-133.

Cao, X. Q., Wang, J. Y., Zhou, L., Chen, B., Jin, Y., and He, Y. W. 2018. Biosynthesis of the yellow xanthomonadin pigments involves an ATPdependent 3-hydroxybenzoic acid: Acyl carrier protein ligase and an unusual type II polyketide synthase pathway. Mol. Microbiol. 110:16-32.

Choi, K. H., and Schweizer, H. P. 2006. mini-Tn7 insertion in bacteria with single attTn7 sites: Example Pseudomonas aeruginosa. Nat. Protoc. 1:153-161.

Dianese, J. C., and Schaad, N. W. 1982. Isolation and characterization of inner and outer membranes of Xanthomonas campestris pv. campestris. Phytopathology 72:1284-1289.

Gaille, C., Kast, P., and Haas, D. 2002. Salicylate biosynthesis in Pseudomonas aeruginosa. Purification and characterization of PchB, a novel bifunctional enzyme displaying isochorismate pyruvate-lyase and chorismate mutase activities. J. Biol. Chem. 277:21768-21775.

Gaille, C., Reimmann, C., and Haas, D. 2003. Isochorismate synthase (PchA), the first and rate-limiting enzyme in salicylate biosynthesis of Pseudomonas aeruginosa. J. Biol. Chem. 278:16893-16898.

Goel, A. K., Rajagopal, L., Nagesh, N., and Sonti, R. V. 2002. Genetic locus encoding functions involved in biosynthesis and outer membrane localization of xanthomonadin in Xanthomonas oryzae pv. oryzae. J. Bacteriol. 184:3539-3548.

Goel, A. K., Rajagopal, L., and Sonti, R. V. 2001. Pigment and virulence deficiencies associated with mutations in the aroE gene of Xanthomonas oryzae pv. oryzae. Appl. Environ. Microbiol. 67:245-250.

Hayward, A. C. 1993. The hosts of Xanthomonas. Pages 1-119 in: Xanthomonas. J. G. Swings and E. L. Civerolo, eds. Springer Netherlands, Dordrecht, The Netherlands. 
He, Y. W., Wu, J., Zhou, L., Yang, F., He, Y. Q., Jiang, B. L., Bai, L., Xu, Y., Deng, Z., Tang, J. L., and Zhang, L. H. 2011. Xanthomonas campestris diffusible factor is 3-hydroxybenzoic acid and is associated with xanthomonadin biosynthesis, cell viability, antioxidant activity, and systemic invasion. Mol. Plant-Microbe Interact. 24:948-957.

Hugouvieux, V., Barber, C. E., and Daniels, M. J. 1998. Entry of Xanthomonas campestris pv. campestris into hydathodes of Arabidopsis thaliana leaves: A system for studying early infection events in bacterial pathogenesis. Mol. Plant-Microbe Interact. 11:537-543.

Jenkins, C. L., and Starr, M. P. 1982. The brominated aryl-polyene (xanthomonadin) pigments of Xanthomonas juglandis protect against photobiological damage. Curr. Microbiol. 7:323-326.

Kovach, M. E., Elzer, P. H., Hill, D. S., Robertson, G. T., Farris, M. A., Roop, R. M. I., and Peterson, K. M. 1995. Four new derivatives of the broad-hostrange cloning vector pBBR1MCS, carrying different antibiotic-resistance cassettes. Gene 166:175-176.

Leyns, F., Marcel De, C., Jean-Guy, S., and De Ley, J. 1984. The Host Range of the Genus Xanthomonas. Bot. Rev. 50:308-356.

Mansfield, J., Genin, S., Magori, S., Citovsky, V., Sriariyanum, M., Ronald, P., Dow, M., Verdier, V., Beer, S. V., Machado, M. A., Toth, I., Salmond, G., and Foster, G. D. 2012. Top 10 plant pathogenic bacteria in molecular plant pathology. Mol. Plant Pathol. 13:614-629.

Moser, R., Aktas, M., and Narberhaus, F. 2014. Phosphatidylcholine biosynthesis in Xanthomonas campestris via a yeast-like acylation pathway. Mol. Microbiol. 91:736-750.

Park, Y. J., Song, E. S., Noh, T. H., Kim, H., Yang, K. S., Hahn, J. H., Kang, H. W., and Lee, B. M. 2009. Virulence analysis and gene expression profiling of the pigment-deficient mutant of Xanthomonas oryzae pathovar oryzae. FEMS Microbiol. Lett. 301:149-155.

Pieterse, C. M. J., Does, D. V., Zamioudis, C., Leon-Reyes, A., and Wees, S. C. M. V. 2012. Hormonal modulation of plant immunity. Annu. Rev. Cell Dev. Biol. 28:489-521.

Poplawsky, A. R., and Chun, W. 1997. pigB determines a diffusible factor needed for extracellular polysaccharide slime and xanthomonadin production in Xanthomonas campestris pv. campestris. J. Bacteriol. 179:439-444.

Poplawsky, A. R., Kawalek, M. D., and Schaad, N. W. 1993. A xanthomonadin-encoding gene cluster for the identification of pathovars of Xanthomonas campestris. Mol. Plant-Microbe Interact. 6:545-552.

Poplawsky, A. R., Urban, S. C., and Chun, W. 2000. Biological role of xanthomonadin pigments in Xanthomonas campestris pv. campestris. Appl. Environ. Microbiol. 66:5123-5127.
Rajagopal, L., Sundari, C. S., Balasubramanian, D., and Sonti, R. V. 1997. The bacterial pigment xanthomonadin offers protection against photodamage. FEBS Lett. 415:125-128.

Starr, M. P., Jenkins, C. L., Bussey, L. B., and Andrewes, A. G. 1977. Chemotaxonomic significance of the xanthomonadins, novel brominated arylpolyene pigments produced by bacteria of the genus Xanthomonas. Arch. Microbiol. 113:1-9.

Stephens, W. L., and Starr, M. P. 1963. Localization of carotenoid pigment in the cytoplasmic membrane of Xanthomonas juglandis. J. Bacteriol. 86: 1070-1074.

Wang, J.-Y., Zhou, L., Chen, B., Sun, S., Zhang, W., Li, M., Tang, H., Jiang, B. L., Tang, J. L., and He, Y. W. 2015. A functional 4-hydroxybenzoate degradation pathway in the phytopathogen Xanthomonas campestris is required for full pathogenicity. Sci. Rep. 5:18456.

Wang, Y., Qian, G., Li, Y., Wang, Y., Wang, Y., Wright, S., Li, Y., Shen, Y., Liu, F., and Du, L. 2013. Biosynthetic mechanism for sunscreens of the biocontrol agent Lysobacter enzymogenes. PLoS One 8:e66633.

Xu, Y., Gao, X., Wang, S. H., Liu, H., Williams, P. A., and Zhou, N. Y. 2012. MhbT is a specific transporter for 3-hydroxybenzoate uptake by Gram-negative bacteria. Appl. Environ. Microbiol. 78: 6113-6120.

Yuan, Z. C., Edlind, M. P., Liu, P., Saenkham, P., Banta, L. M., Wise, A. A., Ronzone, E., Binns, A. N., Kerr, K., and Nester, E. W. 2007. The plant signal salicylic acid shuts down expression of the vir regulon and activates quormone-quenching genes in Agrobacterium. Proc. Natl. Acad. Sci. 104: $11790-11795$.

Zhou, L., Huang, T. W., Wang, J. Y., Sun, S., Chen, G., Poplawsky, A., and He, Y. W. 2013a. The rice bacterial pathogen Xanthomonas oryzae pv. oryzae produces 3-hydroxybenzoic acid and 4-hydroxybenzoic acid via XanB2 for use in xanthomonadin, ubiquinone, and exopolysaccharide biosynthesis. Mol. Plant-Microbe Interact. 26: 1239-1248.

Zhou, L., Li, M., Wang, X. Y., Liu, H., Sun, S., Chen, H., Poplawsky, A., and He, Y. W. 2019. Biosynthesis of coenzyme Q in the phytopathogen Xanthomonas campestris via a yeast-like pathway. Mol. Plant-Microbe Interact. 32:217-226.

Zhou, L., Wang, J. Y., Wang, J., Poplawsky, A., Lin, S., Zhu, B., Chang, C., Zhou, T., Zhang, L. H., and He, Y. W. 2013b. The diffusible factor synthase $\mathrm{XanB2}$ is a bifunctional chorismatase that links the shikimate pathway to ubiquinone and xanthomonadins biosynthetic pathways. Mol. Microbiol. 87:80-93. 